\title{
Teaching Human Anatomy: A Comparison of In-Person and Online Learning Programs
}

\author{
James McKivigan, Noel Guison and Rakhshindah Qureshi
}

\section{ABSTRACT}

Background: The COVID-19 pandemic created challenges in providing anatomy instruction to allied health professions. Human anatomy laboratory classes often rely on human cadavers as instructional material. At some institutions, the anatomical instructional method shifted to online resources. It was essential to compare online methods to those used in the traditional cadaver-based curriculum to determine efficacy. A technique was devised to compare these two approaches. The working hypothesis was that virtual human anatomy models are equally effective to traditional methods in providing anatomy instruction to allied health students.

Methods: Students enrolled in this study participated in a human anatomy course delivered either in-person or virtually via Aclan's Anatomy, NetAnatomy, and Anatomy TV. The instructional design was the same except that the in-person learning group participated in a real-time cadaver anatomy lab, while the virtual learning group utilized online models and simulations. Students were assessed using the same three written tests and three laboratory examinations.

Results: Student demographics and evaluation outcomes were presented, and no significant differences concerning sex or educational program between the two student cohorts were identified. Post hoc testing revealed no statistically significant differences between student cohort and test-type. The three-way interaction between test type, test number, and cohort was not significant.

Conclusions: The findings confirmed the hypothesis. There were no statistically significant differences between the test performance of human anatomy students who received online training versus those who participated in in-person classroom instruction. These results suggest that human anatomy can be taught effectively using an online format.

Keywords: Allied health education, anatomy instruction, cadaver instruction, virtual anatomy.

\section{INTRODUCTION}

The COVID-19 pandemic created numerous challenges for education and the learning process. These challenges were particularly apparent in programs that provided instruction in advanced human anatomy at schools of nursing, medicine, and allied health professions. Traditionally, advanced human anatomy laboratory classes have relied on "hands-on training" with human cadavers as the primary instructional material. The hands-on study of human cadavers is widely regarded as the "gold standard" for medical training and instruction in human anatomy [1]. Human cadaver specimens provide students with direct multisensorial learning of the human body while eliminating the possibility of harming a live person or incurring significant liability resulting from errors made during the educational phase of their career.

Hands-on instruction with human cadaver specimens are respected.
Submitted: August 10, 2021

Published: November 4, 2021

ISSN: 2593-8339

DOI: $10.24018 /$ ejmed.2021.1.6.1011

\section{J. McKivigan*}

School of Physical Therapy, Touro University Nevada, Henderson, Nevada, USA.

(e-mail: jmckivig@touro.edu)

N. Guison

Department of Basic Sciences, Touro University Nevada, Henderson, Nevada, USA.

(e-mail: nguison@ touro.edu)

R. Qureshi

Department of Basic Sciences, Touro University Nevada, Henderson, Nevada, USA.

(e-mail: rqureshi @touro.edu)

*Corresponding Author

requires in-classroom instruction and in-person student participation. Experienced instructors must be continuously physically present during this educational process to assist students who might be nervous or apprehensive about answering questions and correct potential errors. In-person learning also ensures that the remains of the deceased donor

The rapid shift to virtual teaching due to the COVID-19 pandemic (i.e., March 2020) resulted in cataclysmic changes in the instructional programs for human anatomy classes. One certainly cannot provide cadaver specimens for study at home. It is also not feasible to ask students to work alone on these specimens without guidance in an anatomy laboratory. Early in the pandemic, there were few supplies available for COVID-19 testing, and it was nearly impossible to test the cadavers for the presence of the COVID-19 virus. The predominant paradigm in human anatomy instruction quickly shifted from working directly with cadaver specimens to 
working virtually with online models and simulations. It also raised multiple concerns regarding efforts needed to ensure and maintain instruction, learning, and evaluation standards.

Anatomy education fosters a comprehensive understanding of anatomical structures and ultimately aims to enable students to locate and understand their relative positions and varying spatial relationships [2]. In the United States and other nations, current educational practice assumes that hands-on training with human cadaver specimens is the preferred teaching method. With advances in computer programs, it has become possible that virtual training with electronic resources or simulations may be equally effective [3]. Many online nursing schools and training programs for allied health professionals provided virtual, model-based instruction long before the pandemic. This instance is because human cadavers are very expensive and because the students participating in online education are often residing at great distances from the school. Online schools typically cannot require students to attend physical classes in the traditional "brick and mortar" buildings with the classroom facilities necessary to conduct lessons involving human cadavers. As a result, new instructional methods and strategies have been developed.

The use of virtual models for human anatomy training is now accepted as the standard practice among many medical educators [4]. The trend toward virtual learning was observed even before the onset of the COVID-19 pandemic. Traditionally, students studied anatomy using a combination of text-based education, lectures, and cadaver dissection [5]. Many medical professors have noted that the embalmed state of most organs and tissues in human cadavers does not provide accurate representations of those found in a live human subject. There are substantial variations in both the color and texture of anatomical structures in cadavers compared to those in living patients. Cadaver instruction is also hampered by high costs, limited supply, and the comparatively brief period during which cadavers can be suitably preserved [6]. Some of these same arguments (e.g., the limited degree of representation) could also be applied to virtual models and simulations [7].

The use of cadaver specimens to teach human anatomy was adopted during the Age of Enlightenment. There were no other feasible methods for providing students with a direct working knowledge of the human body. Science, technology, and education have developed substantially since that time. Given these developments, it will be essential to compare new methods to those used in the traditional cadaver-based curriculum to determine whether they are equally effective. Educators must devise a means to compare these two approaches and contrast whether virtual learning maintains and upholds the accepted standards of education and promotes student competence in human anatomy.

\section{BACKGROUND}

The working hypothesis is that virtual models of human anatomy are equally, if not more effective, than traditional "hands-on" instruction provided to medical, nursing, and allied health students. The abrupt changes in the higher education system resulting from the COVID-19 crisis provided a "real world" laboratory to test this hypothesis. In this study, two cohorts of allied health science students at Touro University Nevada received instruction in human anatomy were examined. These groups included 40 students engaged in traditional in-person classroom learning before the COVID-19 restrictions were in place and 39 students who received online instruction only with virtual models during the COVID-19 quarantine.

To determine the efficacies of these two methods of instruction, it was ensured that except for the virtual anatomy lab and online lectures, the same course was delivered in both circumstances. The performance of each group was evaluated by the results of three written and three laboratory evaluations. The measures used in these evaluations were reported as percentages; these were statistically assessed using a repeated measures design and modeling using $\mathrm{R}$ (version 3.6.2) to obtain empirical and objective results. As per Hoffman and Roussos (2020), the repeated measures design is appropriate for this type of evaluation [8].

\section{Methodology}

\section{A. Sample}

Students enrolled in this study participated in a human anatomy course delivered either in-person $(n=40)$ or virtually via Aclan's Anatomy, NetAnatomy, and Anatomy TV $(n=39)$. The same instructional design was delivered in both cases because in-person learning involved participation in a real-time gross anatomy lab using cadavers. In contrast, virtual learning utilized virtual models and simulations. Students were graded using the same three written tests and three laboratory evaluations. Performance on all evaluations was evaluated as percentages. The lecture questions were all rated on a uniform determination of the minimum pass level, which provided standardization on a per-item basis.

\section{B. Statistical Analysis}

A repeated measures design was applied to evaluate within-subjects effects of test type (written versus laboratory) and test number (categorically 1-3), the between-subjects effect of the cohort (in-person versus online learning), and interactions between any of the three main effects while controlling for age, sex, and broad program group (i.e., students enrolled in a Bachelor of Science [BSc] versus Bachelor of Arts [BA] / Bachelor of Education [BEd] degree programs). Repeated measures were controlled using a random effect of student numbers through linear mixed modeling. Modeling was performed using $\mathrm{R}$ version 3.6.2, package lme4 (Bates et al., 2015). The normality of residuals was ensured using Q-Q plots. Multicollinearity was negligible using the variance inflation factor.

\section{RESULTS}

\section{A. Sample and Grades}

Student demographics and evaluation outcomes are presented in Table I. A small but significant age discrepancy (1.62 years) between the two cohorts was identified. No significant differences concerning sex or educational program between the two student cohorts were identified. 
TABLE I: DEMOGRAPHICS AND EVALUATION OUTCOMES

\begin{tabular}{|c|c|c|c|c|}
\hline & & In-Person & Online & Difference \\
\hline \multirow{7}{*}{ Program } & Sample Size & 40 & 39 & \multirow{12}{*}{$\begin{array}{c}\mathrm{T}(77)= \\
2.59 \\
P=0.012 \\
\mathrm{X}^{2}=1.57 \\
P=.210 \\
\mathrm{X}^{2}=2.34 \\
P=0.310\end{array}$} \\
\hline & $\begin{array}{l}\text { Age Mean } \\
\text { (SD) }\end{array}$ & $\begin{array}{l}25.30 \\
(2.84)\end{array}$ & $\begin{array}{l}26.92 \\
(2.73)\end{array}$ & \\
\hline & Sex F (\%) & $12(30 \%)$ & $17(44 \%)$ & \\
\hline & Bsc N (\%) & 37 (93\%) & $35(90 \%)$ & \\
\hline & BA N (\%) & $2(5 \%)$ & $4(10 \%)$ & \\
\hline & Bed N (\%) & $1(2 \%)$ & $0(0 \%)$ & \\
\hline & $\begin{array}{l}\text { Written \#1 } \\
\text { Mean } \\
\text { (SD) }\end{array}$ & $\begin{array}{l}79.79 \\
(7.67)\end{array}$ & $\begin{array}{l}78.89 \\
(9.12)\end{array}$ & \\
\hline \multirow{5}{*}{ Grades (\%) } & $\begin{array}{l}\text { Written \#2 } \\
\text { Mean } \\
\text { (SD) }\end{array}$ & $\begin{array}{l}90.91 \\
(7.38)\end{array}$ & $\begin{array}{l}86.73 \\
(7.80)\end{array}$ & \\
\hline & $\begin{array}{c}\text { Written \#3 } \\
\text { Mean } \\
(\mathrm{SD})\end{array}$ & $\begin{array}{l}78.73 \\
(6.61)\end{array}$ & $\begin{array}{l}75.69 \\
(8.52)\end{array}$ & \\
\hline & $\begin{array}{c}\text { Laboratory \#1 } \\
\text { Mean } \\
\text { (SD) }\end{array}$ & $\begin{array}{l}79.94 \\
(8.78)\end{array}$ & $\begin{array}{l}86.99 \\
(8.70)\end{array}$ & \\
\hline & $\begin{array}{c}\text { Laboratory \#2 } \\
\text { Mean } \\
\text { (SD) }\end{array}$ & $\begin{array}{c}84.00 \\
(10.87)\end{array}$ & $\begin{array}{l}81.19 \\
(9.15)\end{array}$ & \\
\hline & $\begin{array}{c}\text { Laboratory \#3 } \\
\text { Mean } \\
(\mathrm{SD}) \\
\end{array}$ & $\begin{array}{l}80.56 \\
(9.79)\end{array}$ & $\begin{array}{l}81.73 \\
(7.39)\end{array}$ & \\
\hline
\end{tabular}

\section{B. Statistical Modeling}

The results obtained by statistical modeling are outlined in Table II. Overall, no significant main effect of the student cohort on evaluation outcomes was found. Specifically, grades on written tests and laboratory evaluations did not differ significantly overall when comparing the results from students who experienced in-person instruction versus those who participated in online classwork.

TABLE II: Statistical Evaluation of Educational Models. The

\begin{tabular}{cccccc}
\multicolumn{5}{c}{ ASTERISK $(*)$ DENOTES P $<0.05$} & \\
\hline \hline Effect & DF1 & DF2 & F & p & \\
\hline \hline Test type (written or & 1 & 385 & 0.86 & 0.354 & \\
laboratory) & & & & & \\
Test number (1, 2, or 3) & 2 & 385 & 34.15 & 0.001 & $*$ \\
Cohort (in-person or online) & 1 & 74 & 0.11 & 0.747 & \\
Age & 1 & 74 & 2.21 & 0.142 & \\
Sex & 1 & 74 & 3.57 & 0.063 & \\
Program & 1 & 74 & 0.32 & 0.571 & \\
Test type $\times$ Test number & 2 & 385 & 27.17 & 0.001 & $*$ \\
Test type $\times$ Cohort & 1 & 385 & 11.82 & 0.001 & $*$ \\
Test number $\times$ Cohort & 2 & 385 & 8.51 & 0.001 & $*$ \\
Test type $\times$ Test number $\times$ & 2 & 385 & 2.11 & 0.123 & \\
Cohort & & & & & \\
\hline \hline
\end{tabular}

Interestingly, a significant effect was observed based on the test number. The scores achieved for the second set of evaluations (both written and laboratory-based) were generally higher than those achieved for the first and third evaluations. The significant interaction between test type and test number reveals that this difference was driven by scores on written evaluations (see Fig. 1, 2 and 3). The effective interaction between student cohort and scores achieved at a given test number was no more pronounced in students receiving in-person instruction than in those participating in online classes.

A significant interaction between student cohort and test type was identified. While students receiving in-person instruction achieved comparatively higher grades on written versus laboratory evaluations, the reverse was true for the online student cohort. However, post hoc testing revealed no statistically significant differences between student cohort and test-type. The three-way interaction between test type, test number, and the cohort was also not significant.

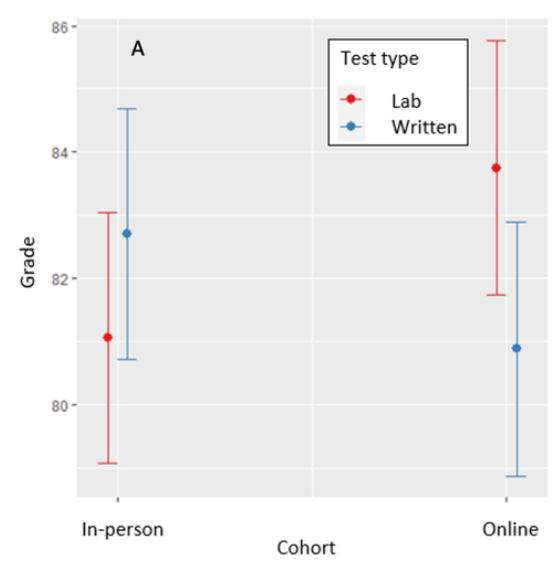

Fig. 1. Comperation of in-person vs. online with test number. The threeway interaction did not reach statistical significance.

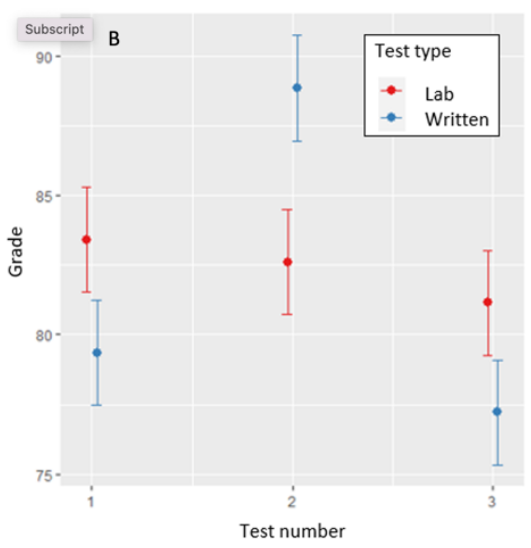

Fig.2. Comperation of test number with test type. The three-way interaction did not reach statistical significance.

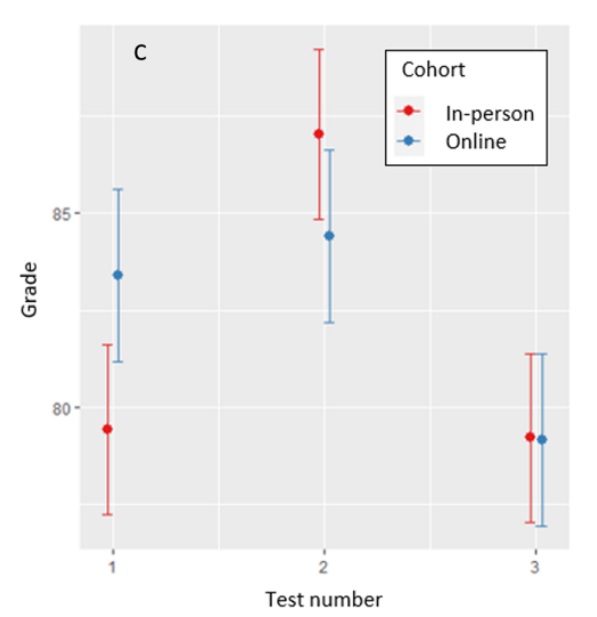

Fig. 3. Comperation of test number with student cohort. The three-way interaction did not reach statistical significance.

\section{Discussion}

This study aimed to compare two different instructional strategies used to teach human anatomy. Specifically, the in- 
person classroom methods that featured instruction with human cadavers versus an online learning system that featured virtual models and simulations were compared. Student cohorts were evaluated for overall differences in performance and relative differences between evaluation types (i.e., written versus laboratory-based examinations). It was hypothesized that the online cohort (i.e., students who received lecture material online) would perform similarly to those attending in-person classes. The findings confirmed this hypothesis, as no statistically significant differences between the two cohorts in overall performance were seen. These results suggest that human anatomy can be taught effectively using an online format. The online design may represent a more cost-effective and accessible alternative to traditional classroom-based learning.

While the two cohorts showed no aggregate differences concerning evaluation type (written or laboratory) and test number (first, second, or third), the initial results suggested that the in-person cohort performed significantly better in the second evaluation than the first and third regardless of evaluation type. By contrast, the online cohort exhibited more consistent performance in the first and second evaluations, with similarly reduced performance at the third evaluation. No significant findings resulted from a direct comparison of the performances of the two cohorts to one another in any three evaluations. While the power of these findings may be limited by sample size, they may reflect trends that might be investigated further. Similarly, while the results suggested trends that distinguished between online and in-person learning and their impact on the specific types and numbers of evaluations, none of these differences reached statistical significance. No conclusions can be drawn regarding the potential for three-way interactions between test type, test number, and student cohort, as differences observed did not achieve statistical significance. This topic is another area that requires further investigation, preferably with larger sample sizes, as each delivery method may have benefits for specific lesson content.

Collectively, the findings suggest that there are no overall differences in performance between the two cohorts. While a deeper investigation into the nuances of specific course content cannot be performed using this dataset because of small sample size, the findings suggest trends that warrant further investigation. Students might also benefit from a combination of in-person and online instruction.

It should also be stressed that the two cohorts were broadly matched in size and demographic characteristics, which were controlled in all analyses. The in-person and online instruction took place at the same time of the year and took place one year apart. The online cohort completed evaluations following the World Health Organization's declaration of COVID-19 as a global pandemic. While one might anticipate that this could have a negative impact on student performance in the online cohort, no discernible differences were identified.

The outcomes of this investigation have significant implications for future educational policy. Among these implications, online education and the use of virtual models and simulations may provide access to numerous students who would be unable to participate in training. The use of high-quality online simulations may ultimately alleviate some of the expenses and concerns regarding the use of human cadaver specimens at some schools. The use of cadavers as a teaching method may prevent some very bright and capable students from considering the healthcare field [9]. Reference [3] found that the use of three-dimensional virtual models may be more effective than traditional cadaver specimens in human anatomy teaching programs. Additional studies will be needed to determine whether more modern models and simulations might replace the long-standing tradition of using cadavers for anatomical instruction for some allied health professional programs.

\section{LiMiTATIONS}

Given the small size of this sample (39-40 students per group), other confounding issues could not be considered. For example, it could not be concluded whether any of the students were uncomfortable with either of the learning formats, particularly with the in-person cadaver dissection. According to [10], the experience of working with a cadaver specimen may provoke past trauma in which a student has witnessed a deceased body or sudden loss of a loved one. Direct interactions with a human cadaver may be overly upsetting for some students. The circumstances presented by COVID-19 also created another complication in this matter. This study did not control for technological capabilities. It may be recognized that some students are more comfortable with online technologies than others, which is another factor that might have an unanticipated impact on the results obtained.

\section{CONCLUSION}

The study has demonstrated no statistically significant differences between the performance of human anatomy students who received online training versus those who participated in in-person cadaveric classroom instruction. Although there are notable limitations associated with the study, the findings support those from earlier peer-reviewed studies, including those with much larger sample sizes, that arrived at similar conclusions. The study results stand in strong support of efforts to develop appropriate online models, simulations, and classroom materials that can be used to support training in human anatomy both now and in the future.

Disclosure statement: The authors have received no funding from any company or organization that would benefit from this article and have no relevant affiliations beyond their academic appointments.

\section{REFERENCES}

[1] J. L. Habicht, C. Kiessling and A. Winkelmann, "Bodies for anatomy education in medical schools: an overview of the sources of cadavers worldwide," Academic Medicine, 93(9), 1293, 2018.

[2] P. G. McMenamin, J. McLachlan, A. Wilson, J. M. McBride, J. Pickering, D. J. Evans and A. Winkelmann, "Do we really need cadavers anymore to learn anatomy in undergraduate medicine?" Medical teacher, 40(10), 1020-1029, 2018.

[3] C. P. Triepels, C. F. Smeets, K. J. Notten, R. F. Kruitwagen, J. J. Futterer, T. F. Vergeldt, and S. M. Van Kuijk, "Does three-dimensional anatomy improve student understanding?" Clinical Anatomy, 33(1), 25-33, 2020. 
[4] Y. Alharbi, M. Al-Mansour, R. Al-Saffar, A. Garman, and A. Alraddadi, "Three-dimensional virtual reality as an innovative teaching and learning tool for human anatomy courses in medical education: A mixed methods study," Cureus, 12(2), 2020.

[5] C. Nesbitt, S. J. Tingle, R. Williams, J. McCaslin, R. Searle, S. Mafeld, and G. Stansby, "A pulsatile fresh frozen human cadaver circulation model for endovascular training: a trial of face validity," Annals of vascular surgery, 46, 345-350, 2018.

[6] B. Preim, and P. Saalfeld, "A survey of virtual human anatomy education systems," Computers \& Graphics, 71, 132-153, 2018.

[7] G. Kovacs, R. Levitan, and R. Sandeski, "Clinical cadavers as a simulation resource for procedural learning," AEM education and training, 2(3), 239-247, 2018.

[8] G. E. Hoffman and P. Roussos "dream: Powerful differential expression analysis for repeated measures designs," Bioinformatics, 37(2), 192-201, 2020.

[9] L. Pelliccia, M. Lorenz, C. E. Heyde, M. Kaluschke, P. Klimant, S. Knopp...N. Hammer, "A cadaver-based biomechanical model of acetabulum reaming for surgical virtual reality training simulators," Scientific reports, 10(1), 1-12, 2020.

[10] J. A. Harris, T. L. Adey, and D. W. McKay, "Is exam performance in anatomy influenced by teaching with prosected cadavers? An evidence-based study," Clinical Anatomy, 33(6), 969-974, 2020. 